\title{
SOFT X-RAY IRRADIATION OF PURE CARBON MONOXIDE INTERSTELLAR ICE ANALOGUES
}

\author{
A. Ciaravella ${ }^{1}$, A. Jiménez-Escobar ${ }^{2}$, G. M. Muñoz Caro $^{2}$, C. Cecchi-Pestellini ${ }^{3}$, R. Candia ${ }^{1}$, \\ S. Giarrusso ${ }^{4}$, M. Barbera $^{5}$, AND A. Collura ${ }^{1}$ \\ ${ }^{1}$ INAF-Osservatorio Astronomico di Palermo, P.za Parlamento 1, 90134 Palermo, Italy; aciaravella @ astropa.unipa.it \\ ${ }^{2}$ Centro de Astrobiología (CSIC-INTA), Carretera de Ajalvir, km 4, Torrejón de Ardoz, 28850 Madrid, Spain \\ ${ }^{3}$ INAF-Osservatorio Astronomico di Cagliari, Strada n.54, Loc. Poggio dei Pini, I-09012 Capoterra (CA), Italy \\ ${ }^{4}$ INAF-Istituto di Astrofisica Spaziale e Fisica Cosmica, Via U. La Malfa 153, I-90146 Palermo, Italy \\ ${ }^{5}$ Dipartimento di Scienze Fisiche \& Astronomiche, Università di Palermo, Sezione di Astronomia, Piazza del Parlamento 1, I-90134 Palermo, Italy \\ Received 2011 November 4; accepted 2011 December 6; published 2012 January 18
}

\begin{abstract}
There is an increasing evidence for the existence of large organic molecules in the interstellar and circumstellar medium. Very few among such species are readily formed in conventional gas-phase chemistry under typical conditions of interstellar clouds. Attention has therefore focused on interstellar ices as a potential source of these relatively complex species. Laboratory experiments show that irradiation of interstellar ice analogues by fast particles or ultraviolet radiation can induce significant chemical complexity. However, stars are sources of intense $\mathrm{X}$-rays at almost every stage of their formation and evolution. Such radiation may thus provide chemical changes in regions where ultraviolet radiation is severely inhibited. After $\mathrm{H}_{2} \mathrm{O}, \mathrm{CO}$ is often the most abundant component of icy grain mantles in dense interstellar clouds and circumstellar disks. In this work we present irradiation of a pure carbon monoxide ice using a soft X-ray spectrum peaked at $0.3 \mathrm{keV}$. Analysis of irradiated samples shows formation of $\mathrm{CO}_{2}, \mathrm{C}_{2} \mathrm{O}, \mathrm{C}_{3} \mathrm{O}_{2}, \mathrm{C}_{3}, \mathrm{C}_{4} \mathrm{O}$, and $\mathrm{CO}_{3} / \mathrm{C}_{5}$. Comparison of X-rays and ultraviolet irradiation experiments, of the same energy dose, shows that $\mathrm{X}$-rays are more efficient than ultraviolet radiation in producing new species. With the exception of $\mathrm{CO}_{2}$, X-ray photolysis induces formation of a larger number of products with higher abundances, e.g., $\mathrm{C}_{3} \mathrm{O}_{2}$ column density is about one order of magnitude higher in the X-ray experiment. To our knowledge this is the first report on X-ray photolysis of $\mathrm{CO}$ ices. The present results show that X-ray irradiation represents an efficient photo-chemical way to convert simple ices to more complex species.
\end{abstract}

Key words: ISM: molecules - methods: laboratory - X-rays: ISM

\section{INTRODUCTION}

Stars are sources of intense X-rays at almost every stage of their formation and evolution, from low-mass brown dwarfs to massive O stars (e.g., Vaiana et al. 1981). In particular, in the first $300 \mathrm{Myr}$ of the life of a solar type star, the high energy component is much larger than vacuum UV emission (Ribas et al. 2005). As a result, circumstellar environments are ionized and heated out to considerable distances, well beyond the reach of the UV radiation field. Such conditions lead to complex gasphase chemistry and gas-grain interactions, favoring otherwise inhibited chemical channels.

Observations have shown that a large variety of molecules exist in the solid phase on dust grains in dense interstellar clouds and circumstellar regions (Gerakines et al. 1999; Ehrenfreund et al. 1999; Dartois et al. 1999; Gibb et al. 2001, 2004; Thi et al. 2002; Boogert et al. 2004; Pontoppidan et al. 2005). $\mathrm{H}_{2} \mathrm{O}$ is generally the most abundant (e.g., Boogert et al. 2008), but significant amounts of other molecules such as $\mathrm{CO}, \mathrm{CO}_{2}$, $\mathrm{H}_{2} \mathrm{CO}$, and $\mathrm{CH}_{3} \mathrm{OH}$ were also detected. Moreover, most of these molecules have also been seen in comets and very recently on asteroidal surfaces (Campins et al. 2010; Rivkin \& Emery 2010). The photo-processing of analogues of such ices to initiate reactions of astronomical interest has almost exclusively been done using ultraviolet (UV) photons. These processes efficiently produce radicals, leading to complex species (e.g., Agarwal et al. 1985; Briggs et al. 1992; Bernstein et al. 1995, 2002; Muñoz Caro et al. 2002; Muñoz Caro \& Schutte 2003; Meierhenrich et al. 2005; Nuevo et al. 2006; Öberg et al. 2010). Some experiments have been also performed exploiting extreme UV sources (e.g., Wu et al. 2002). In such experiments, new molecular species, with respect to vacuum UV experiments, have been synthesized because singly, doubly, multiply charged, and/or electronically excited photo fragments are produced.

Toward most lines of sight where ice absorptions are observed, only water is generally more abundant than $\mathrm{CO}$ in the ice matrix. High resolution spectroscopic observations of solid CO toward a large sample of embedded objects show that solid $\mathrm{CO}$ in interstellar ices may reside in a nearly pure form, segregated from other molecules, as well as in $\mathrm{H}_{2} \mathrm{O}$-rich environments (Ehrenfreund et al. 1996). There is extensive literature on radiation processing of $\mathrm{CO}$ by vacuum UV photolysis and through energetic particle bombardment (e.g., Jamieson et al. 2006 and references therein). Results show that CO is the key to carbon dioxide formation and the precursor of carbon chains and carbon oxide species. UV photodesorption of $\mathrm{CO}$ has been also studied in connection with the problem of the balance of freeze-out timescales in dense cores (Öberg et al. 2007, 2009; Muñoz Caro et al. 2010). The spectra of pure, mixed, and layered $\mathrm{CO}$ and $\mathrm{CO}_{2}$ ices have been investigated by, e.g., van Broekhuizen et al. (2006).

We performed experiments aimed to validate the hypothesis that X-rays play a significant role in the chemistry of interstellar ices. X-ray ice processing is particularly attractive because it may proceed efficiently in regions where UV radiation is severely inhibited. In a preceding work, we presented the results of $0.3 \mathrm{keV}$ photon irradiation of a methanol ice at $8 \mathrm{~K}$ under ultrahigh vacuum conditions, exploiting a weak X-ray source. Despite the low irradiation dose of about $10^{-6}$ photons molecule ${ }^{-1}$, the formation of formaldehyde has been observed (Ciaravella et al. 2010). In this work we present soft X-ray irradiation of a pure carbon monoxide ice, using a 


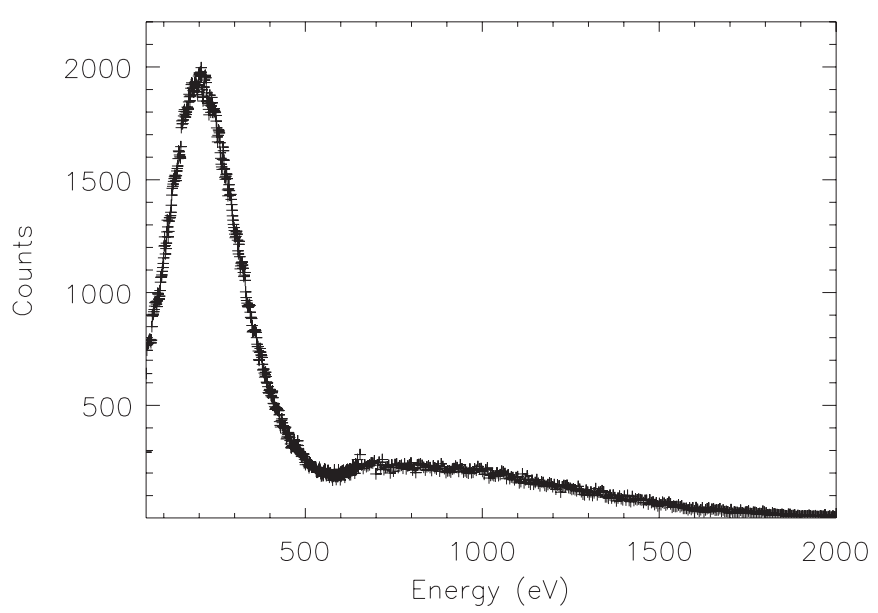

Figure 1. X-ray spectrum of the X-ray source used for the irradiation of the sample. The flux at the ice sample is $6.05 \times 10^{9}$ photons $\mathrm{cm}^{-2} \mathrm{~s}^{-1}$ or $2.29 \times$ $10^{12} \mathrm{eV} \mathrm{cm}^{-2} \mathrm{~s}^{-1}$.

more powerful X-ray photon source. The experiments are described in Section 2 and the results presented in Section 3. We discuss the resulting photo-product inventory and the astrophysical implications in Section 4.

\section{IRRADIATION EXPERIMENTS}

The $\mathrm{CO}$ ice irradiation experiments have been performed using the novel InterStellar Astrochemistry Chamber (ISAC) at the Centro de Astrobiología, Madrid. ISAC is an ultrahigh vacuum setup $\left((2.5-4.0) \times 10^{-11} \mathrm{mbar}\right)$ where an ice sample is made by depositing a gas mixture onto a cold finger from a closed-cycle helium cryostat. Samples can be irradiated or heated to room temperature in a controlled way. The solid sample is monitored by in situ transmittance Fourier Transform Infrared (FTIR) spectroscopy, while the volatile species are detected by quadrupole mass spectroscopy (QMS). We refer to Muñoz Caro et al. (2010) for a detailed description of ISAC. The $\mathrm{CO}$ ice was irradiated using an electron impact $\mathrm{X}$-ray source built at the X-ray Astronomy Calibration and Testing (Barbera et al. 2006) facility of the INAF-Osservatorio Astronomico di Palermo. The source provides an X-ray flux of $6.05 \times 10^{9}$ photons $\mathrm{cm}^{-2} \mathrm{~s}^{-1}$ at the sample position, nearly two orders of magnitude higher than the flux used in our previous experiments (see Ciaravella et al. 2010). Figure 1 shows the spectrum emitted by the source as measured with a Gas Flow Proportional Counter. It includes the $\mathrm{C} \mathrm{K} \alpha$ line and the bremsstrahlung continuum above the absorption edge.

Carbon monoxide (Praxair, 99.998\% purity) was deposited onto a CsI window kept at a temperature of $8 \mathrm{~K}$ during the experiments. The deposition was done at a rate of 0.3 monolayers s ${ }^{-1}$ with a monolayer corresponding to a column density of $1.0 \times 10^{15}$ molecule $\mathrm{cm}^{-2}$.

Several experiments were run with different ice thicknesses and irradiation times. We report here the one with the longest irradiation time, $23 \mathrm{hr}$, as it displays the largest number of photoproducts. The ice column density is $2.4 \times 10^{18}$ molecule $\mathrm{cm}^{-2}$ and has been computed from the $\mathrm{CO}$ infrared absorption band at $2138 \mathrm{~cm}^{-1}$ using the $\mathrm{CO}$ band strength value of $1.1 \times$ $10^{-17} \mathrm{~cm} \mathrm{molecule^{-1 }}$ (Jiang et al. 1975). For a density of $1.0288 \mathrm{~g} \mathrm{~cm}^{-3}$ (Jiang et al. 1975) the corresponding ice thickness is $1.085 \mu \mathrm{m}$ and the total absorbed radiation is $1.2 \times$ $10^{17} \mathrm{eV} \mathrm{cm}^{-2}$. Most of the X-ray photons are absorbed near

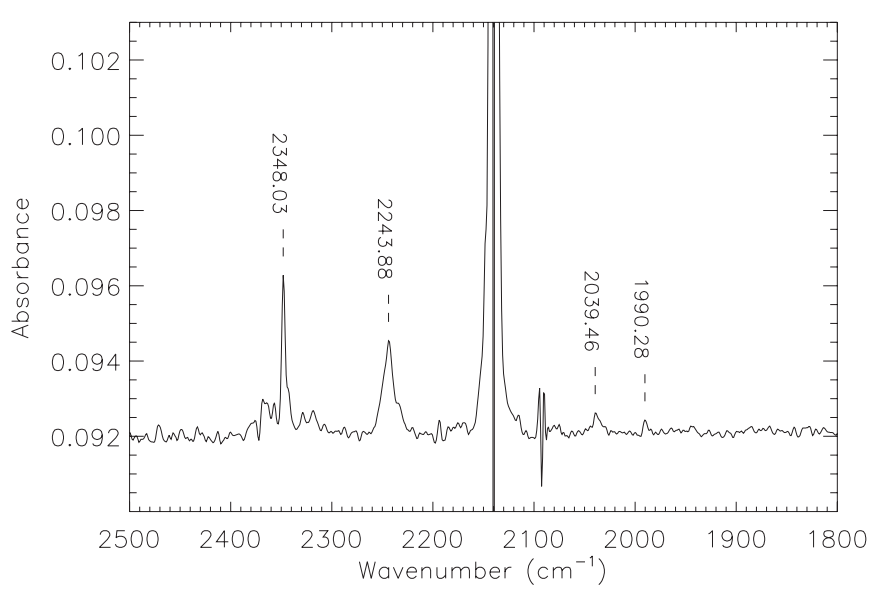

Figure 2. Infrared difference spectrum of $\mathrm{CO}$ ice obtained by subtracting the spectra before and after X-ray irradiation at $8 \mathrm{~K}$.

the absorption edges of carbon $(283 \mathrm{eV})$ and oxygen $(531 \mathrm{eV})$ atoms, with only a small fraction absorbed at higher energies.

After irradiation, ice sublimation was induced by heating at a constant rate of $1 \mathrm{~K}$ minute ${ }^{-1}$. The evolution of the ice was monitored by FTIR spectroscopy in transmittance at a spectral resolution of $1 \mathrm{~cm}^{-1}$, taking spectra before and after the irradiation. Infrared spectra were collected during warmup every $10 \mathrm{~K}$ from $8 \mathrm{~K}$ to $150 \mathrm{~K}$, and every $5 \mathrm{~K}$ from $150 \mathrm{~K}$ to $170 \mathrm{~K}$. The desorbed gas molecules were continuously monitored inside the chamber by QMS.

Blank experiments with no irradiation were performed under the same conditions as the irradiation experiments, keeping the $\mathrm{CO}$ ice inside the chamber for an amount of time equal to the duration of the X-ray irradiation experiments. Then warm-up was started.

A UV irradiation experiment was performed for comparison. The CO ice was irradiated for a total time of 157 minutes using a hydrogen flow discharge lamp that provides a flux of $2.5 \times$ $10^{14}$ photons $\mathrm{cm}^{-2} \mathrm{~s}^{-1}$ at the sample position with an average photon energy of $9.2 \mathrm{eV}$ (Muñoz Caro et al. 2010). The column density of deposited $\mathrm{CO}$ ice in the UV experiment used for comparison was $4.4 \times 10^{17}$ molecule $\mathrm{cm}^{-2}$, accounting for absorption of about $81 \%$ of the incident UV photons (see Muñoz Caro et al. 2010). Infrared spectra were taken at total irradiation times of $30 \mathrm{~s}, 90 \mathrm{~s}, 3$ minutes, 5 minutes, 10 minutes, 30 minutes, 123 minutes, and 157 minutes. The range of total fluence at the sample position was $6.9 \times 10^{16}-2.2 \times 10^{19} \mathrm{eV} \mathrm{cm}^{-2}$. The total absorbed radiation of $1.2 \times 10^{17} \mathrm{eV} \mathrm{cm}^{-2}$ in the X-ray experiment corresponds to approximately $193 \mathrm{~s}$ irradiation in the UV experiment.

\section{RESULTS}

Table 1 lists the products of X-ray irradiation along with their band strengths. The column densities for the products detected in the infrared spectra are also reported. For comparison the column densities obtained from UV irradiation of 3 and 5 minutes are listed as well. Figure 2 shows the infrared difference spectrum obtained by subtracting the ice infrared spectra before and after X-ray irradiation at $8 \mathrm{~K}$. The wavenumber positions of the bands associated with some of the irradiation products are given. The products detected after $\mathrm{X}$-ray irradiation of $\mathrm{CO}$ were $\mathrm{CO}_{2}, \mathrm{C}_{2} \mathrm{O}, \mathrm{C}_{3} \mathrm{O}_{2}, \mathrm{C}_{3}, \mathrm{C}_{4} \mathrm{O}$, and $\mathrm{CO}_{3} / \mathrm{C}_{5}$. The ion current values for $m / z 24,36,40,60,64$, and 68, measured by the QMS as a function of the temperature during warm-up, 

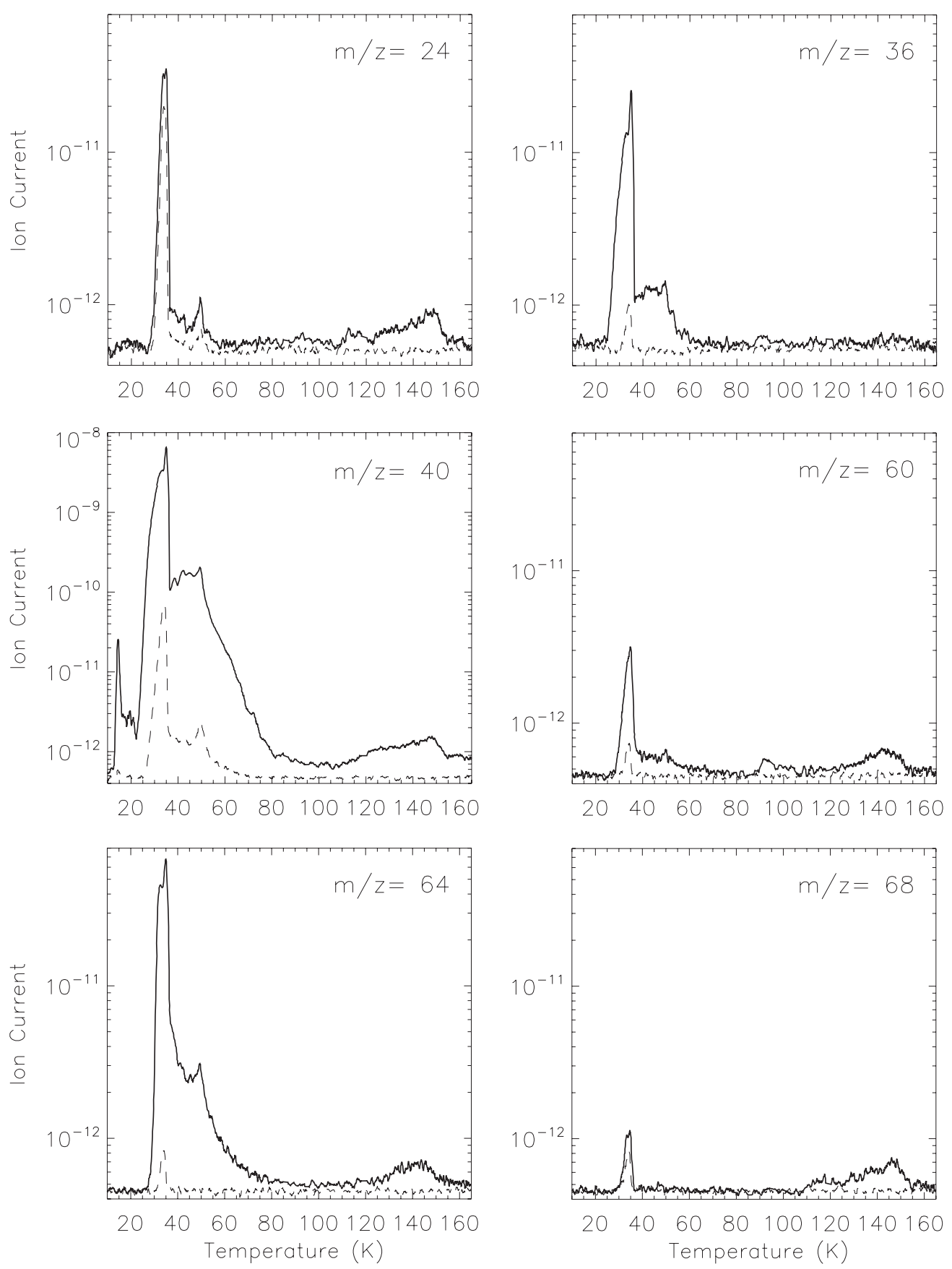

Figure 3. Ion current vs. temperature for $m / z$ 24, 36, 40, 60, 64, and 68 as detected by QMS during warm-up after X-ray irradiation of CO ice. The continuum and dashed lines correspond to the irradiated and blank ice experiments, respectively.

Table 1

CO Ice Irradiation Products in X-ray and UV Experiments

\begin{tabular}{|c|c|c|c|c|c|c|}
\hline \multirow{2}{*}{$\begin{array}{l}v \\
\left(\mathrm{~cm}^{-1}\right)\end{array}$} & \multirow[t]{2}{*}{$m / z$} & \multirow[t]{2}{*}{ Identification } & \multirow{2}{*}{$\begin{array}{c}A \\
\left(\mathrm{~cm} \mathrm{molecule}{ }^{-1}\right)\end{array}$} & \multicolumn{3}{|c|}{$N\left(10^{14}\right.$ molecule $\left.\mathrm{cm}^{-2}\right)$} \\
\hline & & & & X-ray & $\mathrm{UV}\left(3^{m}\right)$ & $\mathrm{UV}\left(5^{m}\right)$ \\
\hline 2348 & 44 & $\mathrm{CO}_{2}$ & $7.6(-17)$ & 4.54 & 19.5 & 27.8 \\
\hline 2244 & 68 & $\mathrm{C}_{3} \mathrm{O}_{2}$ & $1.3(-17)$ & 53.1 & $\ldots$ & $3.3^{\mathrm{a}}$ \\
\hline 2039 & 36 & $\mathrm{C}_{3}$ & $1.0(-16)$ & $\leqslant 1.06$ & $\ldots$ & $\ldots$ \\
\hline 1990 & 40 & $\mathrm{C}_{2} \mathrm{O}$ & $2.4(-17)$ & $\leqslant 1.15$ & $\ldots$ & $\ldots$ \\
\hline$\ldots$ & 60 & $\mathrm{CO}_{3} / \mathrm{C}_{5}{ }^{\mathrm{b}}$ & & & $\ldots$ & $\ldots$ \\
\hline$\ldots$ & 64 & $\mathrm{C}_{4} \mathrm{O}^{\mathrm{b}}$ & & & $\ldots$ & $\ldots$ \\
\hline
\end{tabular}

Notes.

${ }^{a} \mathrm{C}_{3} \mathrm{O}_{2}$ blended with $\mathrm{C}_{3} \mathrm{O}$.

${ }^{\mathrm{b}}$ Detected after X-ray irradiation by QMS. 
are shown in Figure 3. The dashed line in each panel is the ion current for the same $m / z$ in the blank experiment. QMS is more sensitive than FTIR, allowing the detection of smaller concentrations of products. Some species were therefore only revealed by QMS during thermal desorption of the irradiated ice; see Table 1.

The infrared band at $2348 \mathrm{~cm}^{-1}$ indicates that $\mathrm{CO}_{2}$ was produced by X-ray irradiation (Figure 2). Formation of $\mathrm{C}_{2} \mathrm{O}$ is supported by the infrared band at $1990 \mathrm{~cm}^{-1}$ (Figure 2) along with $m / z 40$ detected by QMS during warm-up at $35 \mathrm{~K}$. The infrared band at $2244 \mathrm{~cm}^{-1}$ is associated with $\mathrm{C}_{3} \mathrm{O}_{2}$. The detection of $m / z 68$ by QMS, Figure 3, corresponds to the desorption of $\mathrm{C}_{3} \mathrm{O}_{2}$ around $110-140 \mathrm{~K}$. In the same temperature range we observed desorption of $m / z$ 40, the main fragment of $\mathrm{C}_{3} \mathrm{O}_{2}$ (Figure 3 ). The $m / z 24$ of $\mathrm{C}_{2}$ shows only a slightly higher desorption peak than the blank in the range $35-50 \mathrm{~K}$, which is negligible because this difference is also observed for most $m / z$ values, but a more significant peak at higher temperature between 135 and $150 \mathrm{~K}$. This indicates that $C_{2}$ is mainly produced in the filament of the QMS as a fragment of $\mathrm{C}_{2} \mathrm{O}$ and other photoproducts containing $\mathrm{C}_{2}$ in their chemical structure.

The infrared band of $C_{3}$ at $2039 \mathrm{~cm}^{-1}$ (Jamieson et al. 2006, and references therein) was tentatively detected in the irradiated ice. The formation of $\mathrm{C}_{3}$ by irradiation of $\mathrm{CO}$ is confirmed by the QMS data presented in Figures 3. The desorption of $m / z 36$ only occurs at temperatures below $60 \mathrm{~K}$, and therefore it is not only a fragment of larger molecules desorbing at temperatures above $100 \mathrm{~K}$, confirming that $\mathrm{C}_{3}$ is formed in the ice. However, since $\mathrm{C}_{4} \mathrm{O}, m / z$ 64, also desorbs at low temperature, its decomposition, $\mathrm{C}_{4} \mathrm{O} \rightarrow \mathrm{C}_{3}+\mathrm{CO}$, in the filament region of the QMS could contribute to some of the detected $\mathrm{C}_{3}$ by the QMS.

The $m / z 64$ detection corresponds to $\mathrm{C}_{4} \mathrm{O}$. The infrared band of $\mathrm{C}_{4} \mathrm{O}$ at $1919 \mathrm{~cm}^{-1}$ detected in other experiments (Palumbo et al. 2008; Jamieson et al. 2006) is not observed in our spectra because of the lower sensitivity of the FTIR with respect to the QMS. No infrared bands or $m / z 52$ for tricarbon monoxide, $\mathrm{C}_{3} \mathrm{O}$, were detected. We instead detected $\mathrm{m} / \mathrm{z}$ 60, which is likely associated with $\mathrm{CO}_{3}$, although $\mathrm{C}_{5}$ cannot be excluded based on our current data. While species such as $\mathrm{C}_{6}$ have been revealed in infrared spectra (Jamieson et al. 2006) no detection of the preceding $\mathrm{C}_{5}$ species has been reported in previous irradiation experiments. On the other hand, $\mathrm{CO}_{3}$ has been detected as products of UV irradiation of $\mathrm{CO}_{2}$ (Gerakines et al. 1996) with infrared bands at $976,1067,1883$, and $2044 \mathrm{~cm}^{-1}$. None of these bands were clearly seen in our spectra. From the results of our experiments the $\mathrm{C}_{2} \mathrm{O}, \mathrm{CO}_{2}$, and $\mathrm{C}_{3} \mathrm{O}_{2}$ species are the first products of the irradiation, while the $\mathrm{C}_{3}, \mathrm{C}_{4} \mathrm{O}$ and $\mathrm{CO}_{3} / \mathrm{C}_{5}$ species arise at longer irradiation time. In experiments of proton irradiation of $\mathrm{CO}, \mathrm{C}_{4} \mathrm{O}$ arises later during the irradiation as compared to $\mathrm{C}_{3} \mathrm{O}_{2}$ (Trottier \& Brooks 2004).

$\mathrm{UV}$ irradiation of $\mathrm{CO}$ lead to the formation of $\mathrm{CO}_{2}, \mathrm{C}_{3} \mathrm{O}_{2}$, and $\mathrm{C}_{3} \mathrm{O}$. The most energetic photons emitted by the UV lamp, Ly $\alpha$, only dissociate $\mathrm{CO}$ molecules indirectly by the reaction $\mathrm{CO}^{*}+\mathrm{CO} \rightarrow \mathrm{CO}_{2}+\mathrm{C}$. After $2.5 \mathrm{hr}$ irradiation of optically thick $\mathrm{CO}$ ice, photo-products account for less than $5 \%$ of the $\mathrm{CO}$ band decrease, and the main effect observed is the photodesorption of CO (Muñoz Caro et al. 2010). This is because $\mathrm{CO}_{2}$ formation reaches a maximum after $\sim 15$ minutes of irradiation in our experiments. The resulting column densities for 3 and 5 minute irradiation (bracketing the used X-ray dose) are shown in Table 1 . The abundance of $\mathrm{CO}_{2}$ in the X-ray experiment is lower. But $\mathrm{C}_{3} \mathrm{O}_{2}$, detected after 5 minute irradiation in the UV experiment, has a much lower abundance than in the $\mathrm{X}$-ray experiment. A band at $2248 \mathrm{~cm}^{-1}$ overlaps with the feature of $\mathrm{C}_{3} \mathrm{O}_{2}$ in the UV experiment, but was not detected in the $\mathrm{X}$-ray experiment. This band was attributed to $\mathrm{C}_{3} \mathrm{O}$ (Jamieson et al. 2006 and references therein), but its molecular ion peak at $m / z 52$ was not clearly detected by QMS, suggesting that the $\mathrm{C}_{3} \mathrm{O}$ molecule may be converted to $\mathrm{C}_{3} \mathrm{O}_{2}$ before it desorbs. In addition, the other species detected by QMS in the $\mathrm{X}$-ray experiment, see Table 1, were not observed in the UV experiment. For two of those species, $\mathrm{C}_{3}$ and $\mathrm{C}_{2} \mathrm{O}$, two possible weak bands provide upper limits of their column densities; see Table 1 . We conclude that for the same dose, only $\mathrm{CO}_{2}$ has a higher abundance in the UV experiment; the other products are formed more efficiently by X-ray irradiation.

\section{CONCLUSIONS AND ASTROPHYSICAL IMPLICATIONS}

Soft X-ray irradiation of a $\mathrm{CO}$ ice lead to formation of $\mathrm{CO}_{2}$, $\mathrm{C}_{2} \mathrm{O}, \mathrm{C}_{3} \mathrm{O}_{2}, \mathrm{C}_{3}, \mathrm{C}_{4} \mathrm{O}$, and $\mathrm{CO}_{3} / \mathrm{C}_{5}$. The main products of the $\mathrm{X}$-ray irradiation are common to UV irradiation experiments in the literature. However, for the same irradiation dose, only the $\mathrm{CO}_{2}$ abundance is higher in the UV experiment; the other products are formed more efficiently by $\mathrm{X}$-ray irradiation. The column density of $\mathrm{C}_{3} \mathrm{O}_{2}$ is about one order of magnitude higher in the $\mathrm{X}$-ray experiment and the other species were only detected in the X-ray experiment. Our X-ray experiments provide detectable concentrations of $\mathrm{C}_{4} \mathrm{O}$ and $\mathrm{CO}_{3} / \mathrm{C}_{5} . \mathrm{C}_{4} \mathrm{O}$ has been reported to form in $\mathrm{CO}$ irradiation experiments with high energy particles (Palumbo et al. 2008; Jamieson et al. 2006). $\mathrm{C}_{5}$ formation in ice irradiation experiments was not reported so far. $\mathrm{CO}_{3}$ results from UV irradiation of $\mathrm{CO}_{2}$ ice (e.g., Gerakines et al. 1996).

The highest dosage, $\mathcal{F} \approx 1.2 \times 10^{17} \mathrm{eV} \mathrm{cm}^{-2}$, used in our experiments is about $1-5$ orders of magnitude smaller than those used either in UV (Gerakines et al. 1996; Gerakines \& Moore 2001; Öberg et al. 2007, 2009; Muñoz Caro et al. 2010) or energetic particles experiments (Haring et al. 1984; Gerakines \& Moore 2001; Trottier \& Brooks 2004; Jamieson et al. 2006; Palumbo et al. 2008; Domaracka et al. 2010). Our experimental results may have important implications in a variety of astrophysical environments where X-rays dominate the local radiation density. Such regions are frequently linked to very early stages of star formation, e.g., young stellar objects (Bruderer et al. 2009). To assess the relevance of $\mathrm{X}$-ray irradiation for interstellar chemistry, we compare our experimental X-ray fluence absorbed by the $\mathrm{CO}$ ice with radiation densities in astronomical regions where $\mathrm{X}$-ray sources are embedded.

The X-ray flux incident on a parcel gas located at a distance $r$ from the source is given by

$$
F_{X}(r)=L_{X} \times \frac{f(r)}{4 \pi r^{2}},
$$

where $f(r)$ is an attenuation factor. Setting $f(r) \simeq$ $\left(10^{22} \mathrm{~cm}^{-2}\right) / N_{\mathrm{H}}$, for $N_{\mathrm{H}} \gtrsim 10^{22} \mathrm{~cm}^{-2}$ (Maloney et al. 1996), Equation (1) reads

$$
F_{X} \simeq 5250 \times \frac{L_{X}^{\star}}{N_{\mathrm{H}}^{\star} r_{\mathrm{pc}}^{2}} \mathrm{eV} \mathrm{cm}^{-2} \mathrm{~s}^{-1},
$$

where $L_{X}^{\star}$ is the X-ray luminosity in units of $10^{30} \mathrm{erg} \mathrm{s}^{-1}, N_{\mathrm{H}}^{\star}$ is the hydrogen attenuating column density in units of $10^{22} \mathrm{~cm}^{-2}$, and $r_{\mathrm{pc}}$ the distance from the source in parsec. 
Since X-ray photons are capable of traversing large column densities of gas before being photoelectrically absorbed, they may likely affect the physical and chemical states of the gas in circumstellar disks around solar-type stars. As compared to evolved stars, young solar-type stars are stronger X-ray sources with their flux significantly dominated by harder flaring emission (Favata et al. 2005; Feigelson et al. 2003). Typical quiescent X-ray luminosities of such stars are $L_{X} \approx 10^{30}-10^{31} \mathrm{erg} \mathrm{s}^{-1}$ with X-ray emission peak around $1 \mathrm{keV}$ (Preibisch et al. 2005). During flare activity $\mathrm{X}$-ray luminosity can increase by one order of magnitude, $L_{X} \approx 10^{31}-10^{32} \mathrm{erg} \mathrm{s}^{-1}$, with spectra peaked at energies $\geqslant 2 \mathrm{keV}$ (Favata et al. 2005).

Circumstellar material around such stars is subjected to high $\mathrm{X}$-ray fluxes. Assuming a distance from the central star of $10 \mathrm{AU}$, the distance at which dust temperatures decrease to about $100 \mathrm{~K}$ (Pontoppidan et al. 2007), and a corresponding column density of $N_{\mathrm{H}}=10^{23} \mathrm{~cm}^{-2}$, we obtain X-ray fluxes as low as $2 \times 10^{11} \mathrm{eV} \mathrm{cm}^{-2} \mathrm{~s}^{-1}$ at the minimum of the quiescent phase and as high as $2 \times 10^{13} \mathrm{eV} \mathrm{cm}^{-2} \mathrm{~s}^{-1}$ during the maximum of the flaring phase. At these column densities the ultraviolet radiation is totally inhibited and even the locally generated ultraviolet flux by cosmic-ray induced fluorescence of $\mathrm{H}_{2}$ is several order of magnitudes lower than X-ray fluxes, $F_{\mathrm{UV}} \approx 1 \times 10^{4}-10^{5} \mathrm{eV} \mathrm{cm}^{-2} \mathrm{~s}^{-1}$ (Cecchi-Pestellini \& Aiello 1992; Shen et al. 2004).

In such conditions the highest dosage, $\mathcal{F}$, used in our experiments would require a local irradiation time $t=\mathcal{F} / F_{X} \approx$ 7 days for the minimum value of the quiescent phase and $\approx 0.2$ day for maximum value during the flaring phase. Using as typical ice mantle thickness $10 \mathrm{~nm}$ as in dense clouds, we must rescale the irradiation time by the ratio of the absorbance at $10 \mathrm{~nm}$ and $1 \mu \mathrm{m}$, the ice thickness used in our experiments. Moreover, since X-ray emission from young solar-type stars are peaked at energies higher than the ones used in our experiments, we must also take into account the difference in the absorbances of the ice at those energies. The ratio of the absorbances for a $10 \mathrm{~nm}$ ice as compared to the $1 \mu \mathrm{m}$ are 0.025 at $0.3 \mathrm{keV}, 0.01$ at $1 \mathrm{keV}$ (quiescent young solar-type star), and 0.0094 at $2 \mathrm{keV}$ (flaring young solar-type star). Thus, the dosage used in our experiment would require, for a $10 \mathrm{~nm}$ ice, a local irradiation time $t \approx 6-0.06 \mathrm{yr}$ for a young solar-type star in quiescent phase $\left(L_{X} \approx 10^{30} \mathrm{erg} \mathrm{s}^{-1}\right.$ peaked at $\left.1 \mathrm{keV}\right)$ and for young solartype star in flaring phase $\left(L_{X} \approx 10^{32} \mathrm{erg} \mathrm{s}^{-1}\right.$ peaked at $\left.2 \mathrm{keV}\right)$, respectively.

In conclusion, $\mathrm{X}$-rays emitted by young stars may significantly affect the physical and chemical conditions of the circumstellar material. The results of our experiments suggest that $\mathrm{X}$-rays are potentially important for the understanding of prebiotic chemistry in protoplanetary disks.

We are grateful to the Director of OAPA, Dr. S. Sciortino, for the financial support to our research activity. This work was also financially supported by the Spanish MICINN under project AYA2008-06374 and CONSOLIDER grant CSD200900038. We thank S. Varisco for technical support and Dr. G. Micela for the useful discussion on X-ray emission of young solar-type stars.

\section{REFERENCES}

Agarwal, V. K., Schutte, W. A., Greenberg, J. M., et al. 1985, Orig. Life Evol. Biosph., 16, 21

Barbera, M., Candia, R., Collura, A., et al. 2006, Proc. SPIE, 6266, 62663F

Bernstein, M. P., Dworkin, J. P., Sandford, S. A., Cooper, G. W., \& Allamandola, L. J. 2002, Nature, 416, 401

Bernstein, M. P., Sandford, S. A., Allamandola, L. J., Chang, S., \& Scharberg, M. A. 1995, ApJ, 454, 327

Boogert, A. C. A., Pontoppidan, K. M., Lahuis, F., et al. 2004, ApJS, 154, 359

Boogert, A. C. A., Pontoppidan, K. M., Knez, C., et al. 2008, ApJ, 678, 985

Briggs, R., Ertem, G., Ferris, J. P., et al. 1992, Orig. Life Evol. Biosph., 22, 287

Bruderer, S., Doty, S. D., \& Benz, A. O. 2009, ApJS, 183, 179

Campins, H., Hargrove, K., Pinilla-Alonso, N., et al. 2010, Nature, 464, 1320

Cecchi-Pestellini, C., \& Aiello, S. 1992, MNRAS, 258, 125

Ciaravella, A., Muñoz Caro, G. M., Jiménez Escobar, A., Cecchi-Pestellini, C., et al. 2010, ApJ, 722, L45

Dartois, E., Demyk, K., d'Hendecourt, L., \& Ehrenfreund, P. 1999, A\&A, 351, 1066

Domaracka, A., Seperuelo Duarte, E., Boduch, P., et al. 2010, Nucl. Instrum. Methods Phys. Res. B, 268, 2960

Ehrenfreund, P., Boogert, A. C. A., Gerakines, P. A., et al. 1996, A\&A, 315, L341

Ehrenfreund, P., Kerkhof, O., Schutte, W. A., et al. 1999, A\&A, 350, 240

Favata, F., Flaccomio, E., Reale, F., et al. 2005, ApJS, 160, 469

Feigelson, E. D., Gaffney, J. A., Garmire, G., Hillenbrand, L. A., \& Townsley, L. 2003, ApJ, 584, 911

Gerakines, P. A., \& Moore, M. H. 2001, Icarus, 154, 372

Gerakines, P. A., Schutte, W. A., \& Ehrenfreund, P. 1996, A\&A, 312, 289

Gerakines, P. A., Whittet, D. C. B., Ehrenfreund, P., et al. 1999, ApJ, 522, 357

Gibb, E. L., Whittet, D. C. B., Boogert, A. C. A., \& Tielens, A. G. G. M. 2004, ApJS, 151, 35

Gibb, E. L., Whittet, D. C. B., \& Chiar, J. E. 2001, ApJ, 558, 702

Haring, R. A., Pedrys, R., Oostra, D. J., Haring, A., \& De Vries, A. E. 1984, Nucl. Instrum. Methods Phys. Res., 5, 476

Jamieson, C. S., Mebel, A. M., \& Kaiser, R. I. 2006, ApJS, 163, 184

Jiang, G. J., Person, W. B., \& Brown, K. G. 1975, J. Chem. Phys., 64, 1201

Maloney, P. R., Hollenbach, D. J., \& Tielens, A. G. G. M. 1996, ApJ, 466, 561

Meierhenrich, U. J., Muñoz Caro, G. M., Schutte, W. A., et al. 2005, Chem. Eur. J., 11, 4895

Muñoz Caro, G. M., Meierhenrich, U. J., Schutte, W. A., et al. 2002, Nature, 416, 403

Muñoz Caro, G. M., \& Schutte, W. A. 2003, A\&A, 412, 121

Muñoz Caro, G. M., Jiménez-Escobar, A., Martín-Gago, J. Á., et al. 2010, A\&A, 522,108

Nuevo, M., Meierhenrich, U. J., Muñoz Caro, G. M., et al. 2006, A\&A, 457, 741

Öberg, K. I., Fuchs, G. W., Awad, Z., et al. 2007, ApJ, 662, L23

Öberg, K. I., van Dishoeck, E. F., \& Linnartz, H. 2009, A\&A, 496, 281

Öberg, K. I., van Dishoeck, E. F., Linnartz, H., \& Andersson, S. 2010, ApJ, 718, 832

Palumbo, M. E., Leto, P., Siringo, C., \& Trigilio, C. 2008, ApJ, 685, 1033

Pontoppidan, K. M., Dullemond, C. P., Blake, G. A., et al. 2007, ApJ, 656, 980

Pontoppidan, K. M., Dullemond, C. P., van Dishoeck, E. F., et al. 2005, ApJ, 622, 463

Preibisch, T., Kim, Y.-C., Favata, F., et al. 2005, ApJS, 160, 401

Ribas, I., Guinan, E. F., Gudel, M., \& Audard, M. 2005, ApJ, 622, 680

Rivkin, A. S., \& Emery, J. P. 2010, Nature, 464, 1322

Shen, C. J., Greenberg, J. M., Schutte, W. A., \& van Dishoeck, E. F. 2004, A\&A, 415, 203

Thi, W. F., Pontoppidan, K. M., van Dishoeck, E. F., Dartois, E., \& d'Hendecourt, L. 2002, A\&A, 394, L27

Trottier, A., \& Brooks, R. L. 2004, ApJ, 612, 1214

Vaiana, G. S., Cassinelli, J. P., Fabbiano, G., et al. 1981, ApJ, 245, 163

van Broekhuizen, F. A., Groot, I. M. N., Fraser, H. J., van Dishoeck, E. F., \& Schlemmer, S. 2006, A\&A, 451, 723

Wu, C. Y. R., Judge, D. L., Cheng, B.-M., et al. 2002, Icarus, 156, 456 